\title{
DNA-dependent Protein Kinase-targeting SiDNA DT01
}

National Cancer Institute

\section{Source}

National Cancer Institute. DNA-dependent Protein Kinase-targeting siDNA DT01. NCI

Thesaurus. Code C99165.

A proprietary preparation of small interfering DNA (siDNA) molecules with potential chemo/radiosensitizing activity. By mimicking DNA double strand breaks (DSBs), DNAdependent protein kinase-targeting siDNA DT01 inhibits the non-homologous end joining (NHEJ) process, one of the main DNA repair mechanisms, via binding to and activating DNA-dependent protein kinase (DNA-PK), a core component of the NHEJ complex. DNAPK activation causes hyper-phosphorylation of histone variant H2AX on DNA and results in a different phosphorylated pattern of H2AX upon ionizing radiation treatment. This ultimately interferes with the repair of DNA DSBs during chemo- or radiotherapy, thereby increasing tumor cell death. The enhanced ability of tumor cells to repair DSBs plays a major role in the resistance of tumor cells to chemo- and radiotherapy. 